Jeremy J. Ramsden

\title{
The impacts of nanotechnology
}

\begin{abstract}
It is fairly easy to list the likely technical impacts of nanotechnology. The focus on applications to information processing, energy and health implies that these are the areas in which the greatest impact is foreseen. The most important impacts will, however, be on a broader plane, because nanotechnology epitomizes a deeper, more rational view of the universe, including all the practical aspects of our environment. Nevertheless, it is far from certain that humanity will take up the challenge of the new opportunities, the most potentially disruptive of which is the possibility for everyone to participate in shaping his or her own environment, as much a producer as a consumer. For this reason, those who hold this vision and see such a development as the only alternative offering a sustainable way forward for humanity (in other words for promoting its long-term survival) have a special responsibility to cultivate the vision and its realization.
\end{abstract}

Nanotechnology Perceptions 7 (2011) 28-66

Nonsubscribers: purchase individual article 